\title{
Effects of Hypothalamic Deafferentation on Hormonal Facilitation of Lordosis in Ovariectomized Rats*
}

\author{
Korehito YAMANOUCHI AND Yasumasa ARAI \\ Department of Anatomy, Juntendo University School of \\ Medicine, Bunkyo-ku Tokyo 113, Japan
}

\begin{abstract}
Synopsis
Lordosis response in estradiol benzoate (EB)-progesterone (P) primed castrated female rats was studied after 4 types of deafferentation of the medial hypothalamus. Tests were started 4-5 weeks after the brain surgery and ovariectomy. Anterior deafferentation (half-dome cut) at the level behind the suprachiasmatic nucleus (AD-I) and island isolation of the medial basal hypothalamus ( $\mathrm{MBH}$ island) were highly effective to suppress lordosis response in EB-P primed females. However, the cuts placed dorsal (AD-II) or posterior (AD-III) to the AD-I were without apparent suppressive effect on the EB-P induced display of lordosis. These results suggest that the anterolateral fiber connection with the $\mathrm{MBH}$ may participate in lordosis facilitatory mechanisms in EB-P primed females. When reserpine $(\mathbf{R})$ was injected instead of $P$ to EB-primed rats, $R$ effectively facilitated lordosis response in rats with or without the deafferentation (AD-I or $\mathrm{MBH}$ island). This suggests that the $\mathrm{MBH}$ is not necessary to facilitate lordosis in EB-R primed rats. The site of action of $R$ in the central nervous system for facilitating lordosis may not be the same as that of $P$.
\end{abstract}

Induction of lordosis behavior by ovarian hormones is thought to be the result of the direct action of ovarian steroids on the central nervous system. According to the earlier study of Lisk (1962), the direct implantation of estrogen to the preoptic area (POA) and anterior hypothalamus $(\mathrm{AH})$ facilitates lordosis behavior. Destruction of the $\mathrm{AH}$ resulted in impairment of female sexual behavior (Law and Meagher, 1959; Singer, 1968). Thus, the POA-AH has been believed to play an important role in the regulation of female sexual behavior (see Lsik, 1973). However, from the recent finding that direct implantation of estrogen to the ventromedial nucleus (VM) poten-

\section{Received February 27, 1978.}

* This study was party supported by the grants (No. 212113 and 311411) from the Ministry of Education Science and Culture of Japan. tiated lordosis more effectively than that to the POA-AH, Barfield and Chen (1977) suggested that the VM had a predominant role of lordosis facilitation. This concept is well supported by the result that lesions. of this nucleus eliminated the estrogen-induced display of lordosis (Mathews and Edwards, 1977; Mulsbury et al., 1977). Additionally, this nucleus has been demonstrated to contain significant amounts of estrogen receptors (Stumpf, 1970; Pfaff and Keiner, 1973).

Recently, we have postulated that the dorsal neural inputs to the POA and $\mathrm{AH}$ exert an inhibitory influence on the lordosis mediating mechanisms, based on the result that the surgical removal of the dorsal afferents to these areas effectively facilitated lordosis response (Yamanouchi and Arai, 1977). In the present study, as one step 
to elucidate the relationship between the dorsal inhibitory afferents and the lordosis facilitatory mechanism supposed to be located in the POA-AH and/or VM, the effects of fiber connections between the POA-AH and the medial basal hypothalamus $(\mathrm{MBH})$ on lordosis behavior were examined in estrogen and progesterone or reserpine primed ovariectomized rats.

\section{Materials and Methods}

Female Wistar rats (200-260 g) housed under a controlled photoperiod (14/10 hr light/dark) and temperature $\left(24 \pm 1.4^{\circ} \mathrm{C}\right)$ were subjected to 4 types of hypothalamic deafferentations (see Fig. 1) according to a modified technique of Halász and Gorski (1967).
A Halász knife, having a $1.4 \mathrm{~mm} \times 2.0 \mathrm{~mm}$ blade, was lowered to the base of the brain through the sagittal sinus at a point $1.5 \mathrm{~mm}$ posterior to the bregma and was rotated for $180^{\circ}$. anteriorly (anterior deafferentation type I, AD-I). A similar type of half-dome cut was made at the level $1.5 \mathrm{~mm}$ dorsal to the location of the AD-I (anterior deafferentation type II, AD-II). Another half-dome cut was also made at the level $1.0 \mathrm{~mm}$ posterior to the AD-I (anterior deafferentation type III, AD-III). In order to interrupt all afferents to the $\mathrm{MBH}$, the knife was turned $90^{\circ}$ to the left at the same location of the AD-I and moved $2.5 \mathrm{~mm}$ posteriorly. After completing the lateral cut, the knife was rotated for $180^{\circ}$. Then, the knife was moved $2.5 \mathrm{~mm}$ anteriorly and turned back to the left $90^{\circ}$ to complete the island isolation of the $\mathrm{MBH}(\mathrm{MBH}$ island). All

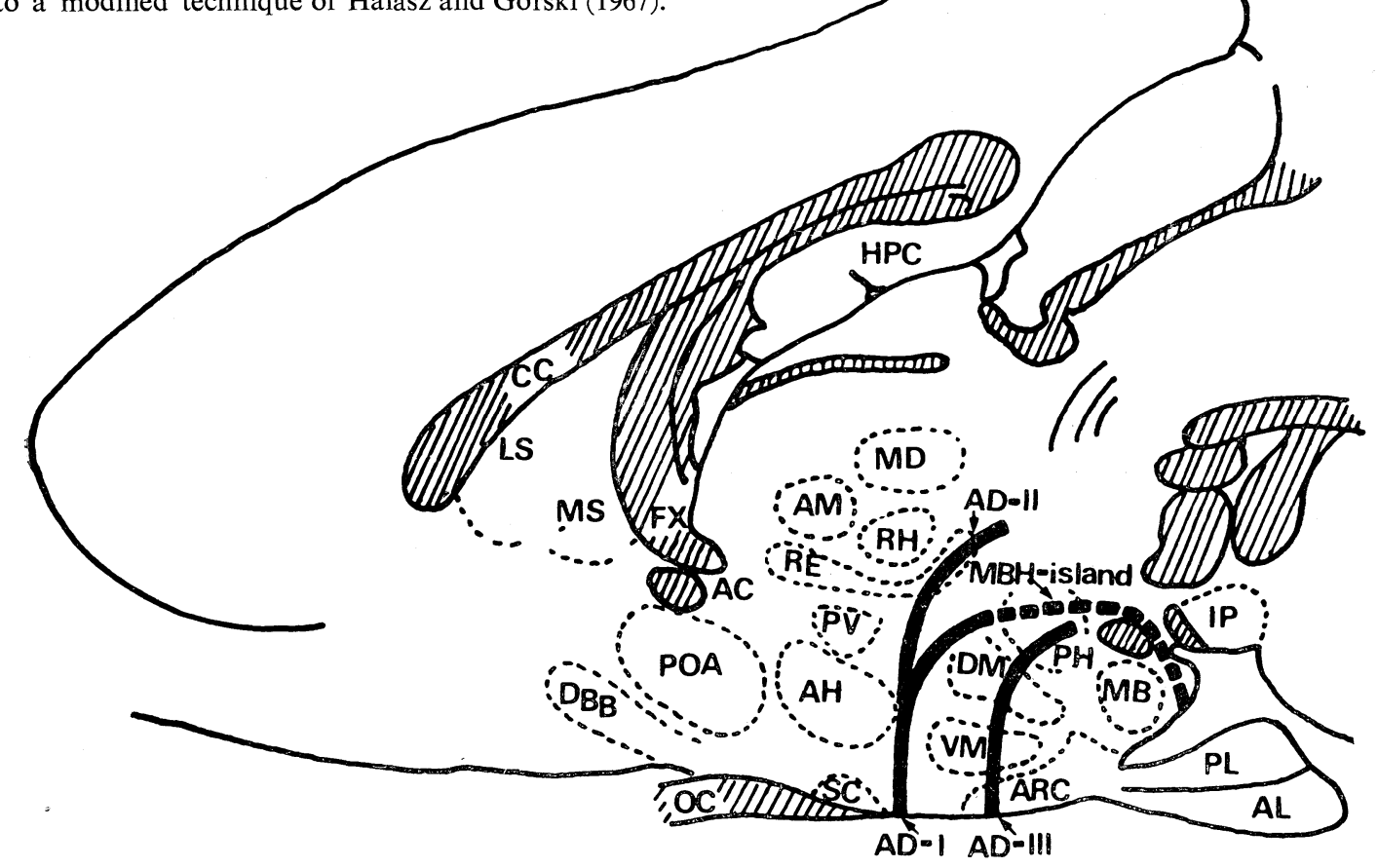

Fig. 1. Schematic representation of the surgical cuts in midsagittal section. AD-I: anterior deafferentation type I, AD-II : anterior deafferentation type II, AD-III: anterior deafferentation type III, MBH-island: island isolation of the medial basal hypothalamus (AD-I and broken line).

AC: anterior commissure, AP: anterior lobe of pituitary, AM: anteromedial thalamic nucleus, ARC: arcuate nucleus, CC: corpus callosum, DBB: nucleus and diagonal band of Broca, DM: dorsomedial nucleus, FX: fornix, HPC: Hippocampus, IP : interpeduncular nucleus, LS : lateral septal nucleus, MB: mammillary body, MD: mediodorsal thalamic nucleus, MS: medial septal nucleus, OC: optic chiasma, PH: posterior hypothalamic nucleus, PL: posterior lobe of pituitary, POA : preoptic area, PV : paraventricular nucleus, RE: nucleus reuniens, RH : nucleus rhomboideus thalami, SC: suprachiasmatic nucleus, VM: ventromedial nucleus. 
female rats receiving the deafferentation were castrated at the same time. A number of females without brain surgery were castrated as controls.

Four to five weeks after the operation, a series of behavioral tests was started at 2 week intervals. At the first test, the animals were injected with $2 \mu \mathrm{g}$ estradiol benzoate (EB, dissolved in $0.1 \mathrm{~m} l$ sesame oil) for 3 successive days and with $0.5 \mathrm{mg}$ progesterone ( $\mathbf{P}$, in $0.1 \mathrm{ml}$ oil) $5-7 \mathrm{hr}$ prior to the behavioral test on the fourth day. At the second test, the dose of EB was increased to $10 \mu \mathrm{g}$. The animals were treated similarly and tested following P. Two weeks after the second test, about half of the animals were subjected to another series of experiments. The animals were treated with $2 \mu \mathrm{g}$ EB for 3 successive days and then injected with $1 \mathrm{mg} / \mathrm{kg}$ reserpine $(\mathbf{R}$, Serpasil, CIBA) 5-7 hr before the test on the fourth day. Again, 2 weeks later, the animals were tested in a similar manner following EB-P priming, increasing dose of $\mathrm{R}$ to $2 \mathrm{mg} / \mathrm{kg}$. A lordosis quotient (LQ, ratio: numbers of lordosis/10 mounts $\times 100$ ) was computed for each animal. The precise localization of the cuts was examined histologically in each brain.

\section{Results}

The results are summarized in Figs. 2 and 3. Daily injections of $2 \mu \mathrm{g}$ EB for 3 days and subsequent injection of $0.5 \mathrm{mg} \mathrm{P}$ induced high levels of lordosis behavior in castrated control females. All of 12 females of this group showed lordosis and the mean LQ was $82.5 \pm 4.3$. In contrast, only 4 out of 14 animals in $\mathrm{MBH}$-island group displayed lordosis and the mean LQ of the group was $8.6 \pm 3.9$. A similar low level of receptivity was observed in AD-I group. Five out of 15 animals of the group showed lordosis (LQ: 17.1 \pm 7.3 ). The incidence of lordosis and the LQ in these two groups were significantly lower than that

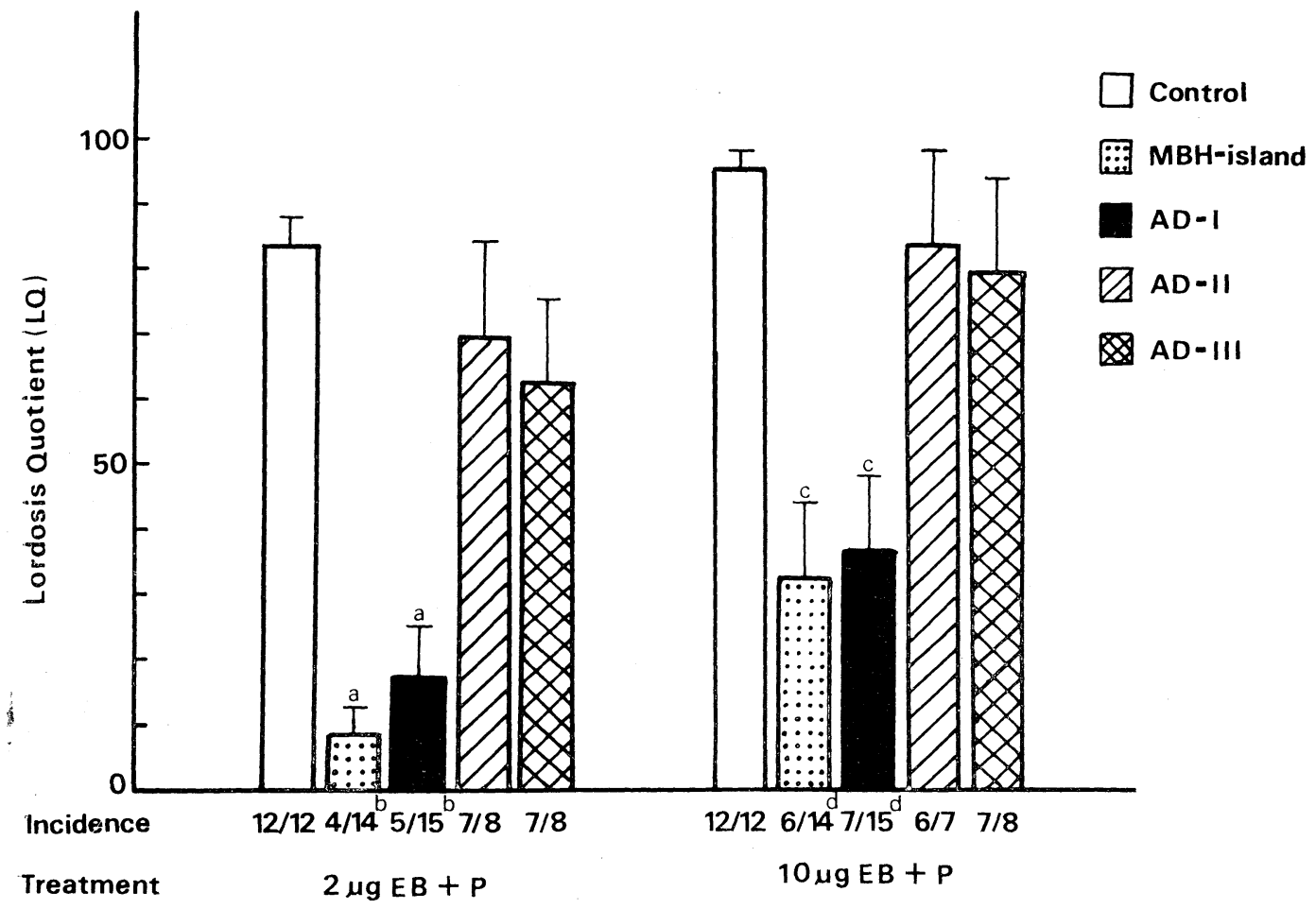

Fig. 2. Effect of hypothalamic deafferentation on induction of lordosis and LQ in EB-P primed ovariectomized rats.

a: $\mathrm{P}<0.005$ vs control or AD-II, $\mathrm{P}<0.01$ vs AD-III $(t$-test).

b: $\quad \mathrm{P}<0.005$ vs control, $\mathrm{P}<0.05$ vs AD-II or AD-III ( $X^{2}$ test with Yates' correction).

c: $\mathrm{P}<0.005$ vs control, $\mathrm{P}<0.025$ vs $\mathrm{AD}$-III, $\mathrm{P}<0.01$ vs $\mathrm{AD}$-II ( $t$-test).

$\mathrm{d}: \quad \mathrm{P}<0.01$ vs control ( $X^{2}$ test with Yates' correction). 
in control and other 2 experimental groups. Rejection behaviors, such as kicking and fighting against the male, were frequently seen in $\mathrm{MBH}$-island $\mathrm{AD}-1$ rats. When a half-dome cut was made more dosal (ADII) or more posterior (AD-III) to the location of the AD-I, 7 out of 8 rats in each group displayed lordosis (LQ: $68.8 \pm 14.8$, $62.5 \pm 12.1$, respectively). When the dose of EB was increased to $10 \mu \mathrm{g}$, all of 12 control rats, 6 out of 7 AD-II rats and 7 out of 8 AD-III rats displayed lordosis (LQ: $95.0 \pm 2.3,82.9 \pm 13.9$ and $78.8 \pm 14.2$, respectively). However, only 6 out of 14 MBH-island rats and 7 out of 15 AD-1 rats exhibited lordosis behavior (LQ: 32.1 \pm 11.4 and $36.7 \pm 11.2$, respectively).
As shown in Fig. 3, when $\mathrm{R}$ was injected instead of $P, R$ effectively induced lordosis behavior in EB-primed control females. The mean LQ of this group was $55.7 \pm 14.6$ when treated with $1 \mathrm{mg} / \mathrm{kg} \mathrm{R}$ in combination with $2 \mu \mathrm{g}$ EB. In MBHisland and AD-I groups, $R$ was also effective. Six out of 8 rats and 9 out of 11 rats showed lordosis, respectively. The mean LQs of these two groups were $63.8 \pm 14.3$ and $50.0 \pm 9.7$, respectively. When the dose of $\mathrm{R}$ was increased to $2 \mathrm{mg} / \mathrm{kg}$, all of 6 $\mathrm{MBH}$-island rats and 9 out of 11 AD-I rats showed lordosis and the mean LQs were $96.7 \pm 3.3$ and $70.9 \pm 12.2$, respectively. These were not statistically different from the incidence and LQ $(77.1 \pm 11.3)$ of control rats.

: MBH-island
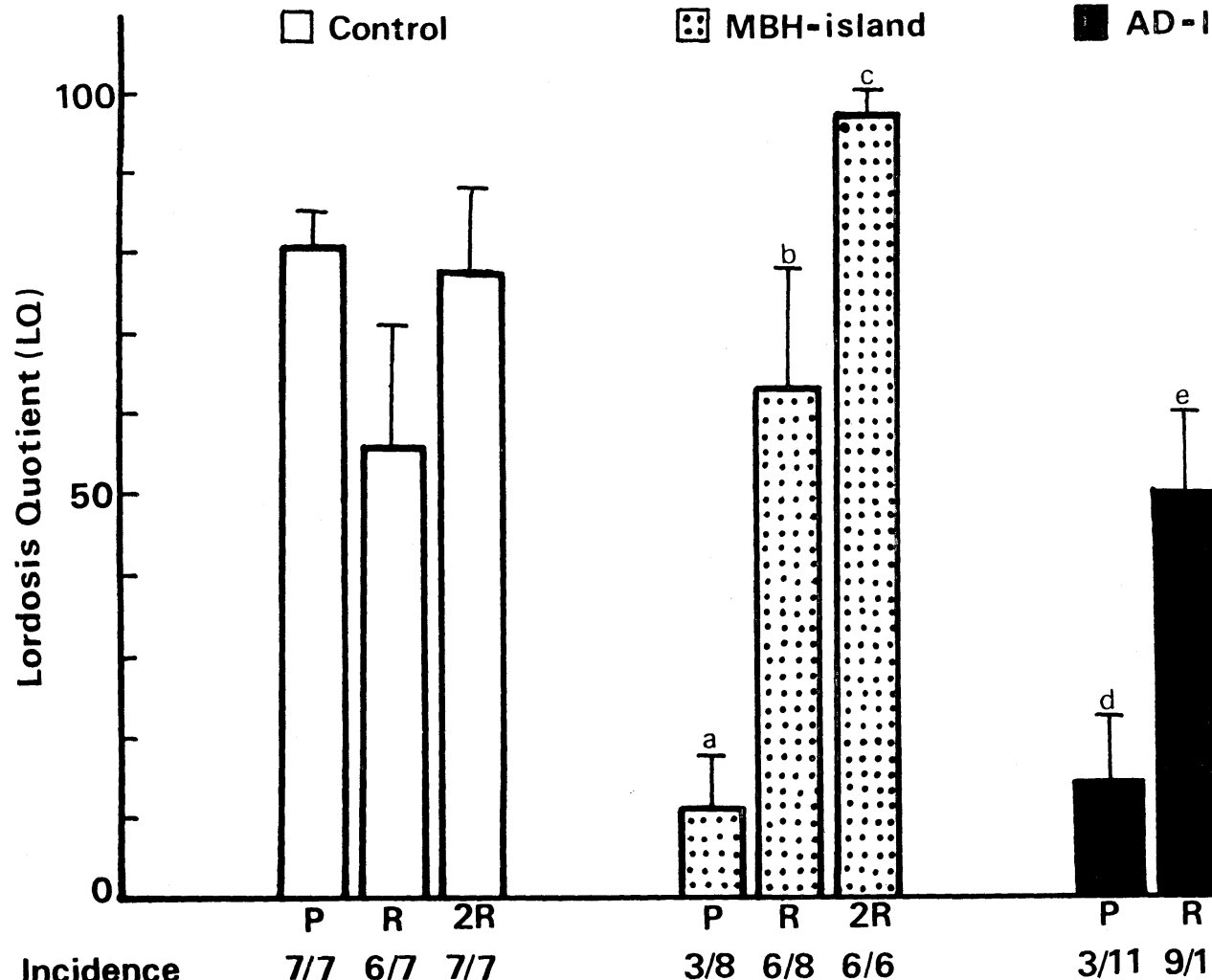

Incidence

Fig. 3. Effect of reserpine on lordosis in control and experimental female rats primed with $2 \mu \mathrm{g}$ EB. $\mathrm{P}: 0.5 \mathrm{mg}$ progesterone, $\mathrm{R}: 1 \mathrm{mg} / \mathrm{kg}$ reserpine, $2 \mathrm{R}: 2 \mathrm{mg} / \mathrm{kg}$ reserpine.

$\mathrm{a}: \mathrm{P}<0.005$ vs b or c, $\mathrm{d}: \mathrm{P}<0.01$ vs e, $\mathrm{P}<0.005$ vs $\mathrm{f}(t$-test $)$. 
Microscopic examinations of the brain showed that the $\mathrm{MBH}$-island included the basal part of the anterior hypothalamic area, the arcuate nucleus, the VM, the dorsomedial nucleus and the mammillary nuclear complex. In some $\mathrm{MBH}$-island females, the cut of one lateral side did not reach perfectly to the base of the brain. However, there was no obvious difference in the results between such rats and rats with complete island. The AD-I was located posterior to the suprachiasmatic nucleus and extended dorsally the paraventricular nucleus. This cut was almost identical with the frontal part of the $\mathrm{MBH}$ island. The VM was separated by the ADIII at the middle level of the nucleus. The posterior part of the VM and the most part of the arcuate nucleus were located behind the AD-III.

\section{Discussion}

The results of the present study clearly show that the anterolateral fiber connections with the $\mathrm{MBH}$ may participate in the lordosis facilitating mechanisms, because the MBH-island and AD-I were effective to suppress lordosis response in EB$P$ primed ovariectomized rats but the ADII failed to do it. This is well consistent with a recent concept proposed by several investigators (Dörner et al., 1968; Mathews and Edwards, 1977; Malsbury et al., 1977; Barfield and Chen, 1977) that the VM plays an important role in the hormonal induction of lordosis behavior. In this context, the effect of the AD-I and MBHisland could be interpreted to interrupt the neural pathways involved in lordosis facilitation entering or leaving anterolaterally the VM region. According to recent neuroanatomical studies (Szentágothai et al., 1968; Conrad and Pfaff, 1976; Saper et al., 1976), these anterolateral inputs and outputs of the VM region were thought to be related not only with the lower brain stem but also with the POA and limbic system. The failure of the ADIII to eliminate lordosis response in EB-P primed females may indicate that the anterior portion of the VM is important for the lordosis facilitating mechanism. Recently, Barfield and Chen (1977) have postulated that the VM is a primary site of estrogenic action to facilitate lordosis. However, the fact that the suppressive effect of the MBH-island and AD-I could effectively be reversed by $R$ injection in the present study may suggest that the $\mathrm{MBH}$ does not play an essential role in facilitating lordosis in EB-R primed rats. $R$ itself has been reported to exert no facilitatory action on lordosis without estrogen priming (Meyerson, 1964a). These suggest together that the minimal estrogen-sensitive component for the display of lordosis may be located other than in the $\mathrm{MBH}$, although the VM must be one of the critical sites for the hormonal facilitation of lordosis behavior.

POA lesion (Powers and Valenstein, 1972 : Christensen et al., 1977) as well as transection of the dorsal afferents to the POA-AH (Yamanouchi and Arai, 1977) has been demonstrated to potentiate lordosis behavior. These results suggest that the dorsal inputs to the hypothalamus exert an inhibitory influence on the lordosis mediating mechanisms in the hypothalamus. Since the AD-I or MBH-island suppressed EB-P induced lordosis response in the present study, the VM does not seem to be a direct focus of this inhibitory influence. The possible relay station of neural signals to transfer from inhibitory to facilitatory may be proposed to exist somewhere anterior to the AD-I or $\mathrm{MBH}$, presumably in the $\mathrm{AH}$ which has been suggested to be one of the hypothalamic facilitatory centers for lordosis by earlier investigators (Law and Meagher, 1958; Singer, 1968; Lisk, 1973). However, there seems to be 
still not sufficient evidence to demonstrate a clear relationship between the dorsal inhibitory system and the VM facilitation system in the regulation of lordosis behavior.

Regarding the mode of action of $\mathrm{R}$ on the lordosis facilitation, it has been suggested that CNS depressants influence lordosis by stimulating the release of ACTH to increase the secretion of adrenal $P$ (Paris et al., 1970; Larsson et al., 1974). However, Lengvári and Halász (1972) have shown that the release of ACTH does not change significantly before and after $\mathrm{R}$ injection in the rats with $\mathrm{MBH}$-island or AD. In the present study, EB-primed $\mathrm{MBH}-$ island or AD-I rats failed to respond to $P$ with lordosis but showed a high level of sexual receptivity following $\mathrm{R}$ injection. Therefore, it is highly probable that $R$ facilitate lordosis by acting directly on the lordosis mediating neural mechanisms somewhere outside of the MBH. This is in agreement with earlier findings of Meyerson (1964 a,b). The possible mode or site of action of $\mathrm{R}$ for facilitating lordosis may be the same as that of $P$. Further study is required to clarify these points.

\section{References}

Barfield, R. J. and J. J. Chen (1977). Endocrinology 101, 1716.

Christensen, L. W., D. M. Nance and R. A. Gorski (1977). Brain Res. Bull. 2, 137.

Conrad, L. C. A. and D. W. Pfaff (1976). J. Comp. Neurol. 169, 221.

Dörner, G., F. Döcke and S. Moustafa (1968). J. Reprod. Fert. 17, 583.

Halász, B. and R. A. Gorski (1967). Endocrinology $80,608$.

Larsson, K., H. H. Feder and B. R. Komisaruk (1974). Pharmacol. Biochem. Behav. 2, 685.

Law, T. and W. Meagher (1958). Science 128, 1626.

Lengvári, I. and B. Halász (1972). J. Neur. Trans. 33, 289.

Lisk, R. D. (1962). Amer. J. Physiol. 203, 493.

Lisk, R. D. Handbook of Physiology, Sec. 7, Vol. II. Greep, R. O. (ed.) Williams and Wilkins, Baltimore, p. 223 (1973).

Malsbury, C. W., L.-M. Kow and D. W. Pfaff (1977). Physiol. Behav. 19, 223.

Mathews, D. and D. A. Edwards (1977). Physiol. Behav. 19, 319.

Meyerson, B. J. (1964a). Psychopharmacol. 6, 210.

Meyerson, B. J. (1964b). Arch. Int. Pharmacodyn. $150,4$.

Paris, C. A., J. A. Resko and R. W. Goy (1971). Biol. Reprod. 4, 23.

Pfaff, D. W. and M. Keiner (1973). J. Comp. Neurol. 151, 121.

Powers, B. and E. S. Valenstein (1972). Science 175, 1003.

Saper, C. B., W. Swanson and W. M. Cowan (1976). J. Comp. Neurol. 169, 409.

Singer, J. J. (1968). J. Comp. Physiol. Psychol. 66, 738 .

Stumpf, W. E. (1970). Amer. J. Anat. 129, 207.

Szentágothai, J., B. Flerkó, B. Mess and B. Halász Hypothalamic Control of the Anterior Pituitary, Akademiai Kiado, Budapest (1968).

Yamanouchi, K. and Y. Arai (1977). Brain Res. 127, 296. 